\section{Optimization and Troubleshooting in PCR}

\author{
Kenneth H. Roux \\ Department of Biological Science, \\ Florida State University, \\ Tallahassee, Florida 32306-3050
}

\section{BASIC CONSIDERATIONS}

The use of PCR to generate large amounts of a desired product can be a double-edged sword. Failure to amplify a sample under optimum conditions can lead to the generation of multiple undefined and unwanted productseven to the exclusion of the desired product. At the other extreme, no product may be amplified. A typical response at this point is to vary one or more of the many parameters that are known to contribute to primer-template fidelity and primer extension. High on the list of optimization variables are $\mathbf{M g}^{2+}$ concentrations, buffer $\mathrm{pH}$, and cycling conditions. With regard to the last, the annealing temperature is most important. The situation is complicated further by the fact that some of the variables are quite interdependent. For example, because dNTPs directly chelate a proportional number of $\mathrm{Mg}^{2+}$ ions, an increase in the concentration of dNTPs decreases the concentration of free $\mathrm{Mg}^{2+}$ available to influence polymerase function.

\section{ENHANCING AGENTS}

Various additives such as DMSO (2\%-5\%), PEG 6000 (5\%-15\%), glycerol (5\%-20\%), nonionic detergents, and formamide (5\%) can also be incorporated into the reaction to increase specificity. ${ }^{(1,2)}$ Some reactions may amplify only in the presence of such additives. ${ }^{(1)}$ Several optimization kits incorporating these and other enhancing agents, and a variety of buffers, are currently being marketed (e.g., by Continental Laboratory Products, Invitrogen, Perkin-Elmer Cetus, and Stratagene). Additional discussion of PCR optimization and contamination avoidance considerations can be found in Ref. 2 .

\section{MATRIX ANALYSES}

The basic challenge is to devise an optimization strategy that is efficient in both time and cost. A full matrix analysis in which several values for each of the variables are tested in combination with each of the other variables can quickly become overwhelmingly cumbersome and costly. The size of the matrix can be pared down significantly by application of the Taguchi method, ${ }^{(3)}$ in which several key variables are altered simultaneously. ${ }^{(4)} \mathrm{A}$ more typical strategy is to run a simple matrix analysis focused on those parameters most likely to have the greatest impact on hybridization fidelity (i.e., $\mathrm{Mg}^{2+}$ concentration and annealing temperature).

\section{$\mathrm{Mg}^{2+}$ CONCENTRATION}

$\mathrm{Mg}^{2+}$ concentration is the easiest to manipulate because all concentration variations can be run in separate tubes simultaneously. Suppliers of Taq polymerase now provide $\mathrm{MgCl}_{2}$ solution separate from the rest of the standard reaction buffer to simplify its adjustment. A typical two-step optimization series might first include $\mathrm{Mg}^{2+}$ at $0.5 \mathrm{~mm}$ increments from 0.5 to $5.0 \mathrm{mM}$ and, after the range is narrowed, at several 0.2 or $0.3 \mathrm{~mm}$ increments.

\section{ANNEALING TEMPERATURE}

Optimization of annealing temperature begins with calculation of the melting temperatures $\left(T_{\mathrm{m}}\right)$ of the primer-template pairs by one of several methods, the simplest being $T_{m}=(G+C) 4+(A+T) 2$. A single base mismatch lowers the $T_{\mathrm{m}}$ by $\sim 5^{\circ} \mathrm{C}$. More complex formulas can be used also, ${ }^{(5,6)}$ but in practice, because the $T_{\mathrm{m}}$ is affected variously by the individual buffer components and even the primer and template concentrations, any calculated $T_{\mathrm{m}}$ value should be regarded as an approximation. Several reactions run at temperature increments $\left(2-5^{\circ} \mathrm{C}\right)$ straddling a point $5^{\circ} \mathrm{C}$ below the calculated $T_{\mathrm{m}}$ will give a first approximation of the optimum annealing temperature for a 
given set of reaction conditions. It should be noted that some primers, for reasons that are not entirely apparent, are refractory to optimization. ${ }^{(7)}$ One possible explanation may be that unique characteristics of the target amplicon give a $T_{\mathrm{m}}$ above the temperature of the denaturation cycle segment. ${ }^{(8)} \mathrm{In}$ such situations, it may be more time and cost efficient simply to design a second set of primers that hybridize to neighboring DNA.

\section{PRIMER DESIGN}

Ideally, PCR primers should have a $40-60 \%$ GC content and a 3 '-terminal "G/C clamp" (at least one or two 3' G's and/or C's); be similar in size (18-25 bases), $T_{\mathrm{m}}$ values, and nucleotide ratios; and be free of repetitive motifs, palindromes, excessive degeneracy, and long stretches of polypurines or polypyrimidines. ${ }^{(8)}$ Fortunately, deviation from any or all of these guidelines is tolerable but may necessitate greater attention to other optimization parameters. Several useful computer programs have been developed to aid in efficient primer design (OLIGO, National BioSciences; GeneWorks and PCGene, Intelligenetics).

\section{CYCLE NUMBER, REAMPLIFICATION, AND PRODUCT SMEARINC}

Increasing the number of cycles may enhance an anemic reaction, but this modification can also lead to the generation of spurious bands and to smears composed of high molecular weight products rich in single-stranded DNA. ${ }^{(9)}$ Similar smearing can occur under normal conditions if the level of starting template is too high, as often occurs in attempts to reamplify from a previous PCR. A general rule of thumb is to use $1 \mu \mathrm{l}$ of a $1: 10^{4}-10^{5}$ dilution of a PCR if a gel band is detectable.

\section{PIPETTING AND THERMAL CYCLER VARIABLES}

To minimize pipetting variables, a master mix containing all of the reactants except the Taq polymerase can be made in advance and stored at $4^{\circ} \mathrm{C}$ for the several days (or even longer) that it might take to run the reactions at each of the selected annealing temperatures. If multiple thermal cyclers are available, it may be tempting to run the reactions concurrently, but small differences in calibration and performance among machines could render the results useless, ${ }^{(10,11)}$ particularly for marginal reactions. Also, when running multiple sequential reactions, it is prudent to use the same wells on the sample block to avoid possible well-to-well variations. ${ }^{(10,11)}$ At least one manufacturer of thermal cyclers (Stratagene) offers a machine that can run eight different temperature profiles simultaneously in the same block to aid in annealing temperature optimization.

\section{TOUCHDOWN PCR}

Touchdown (TD) PCR represents a fundamentally different approach to PCR optimization. ${ }^{(12)}$ Rather than multiple reaction tubes, each with different reagent concentration and/or cycling parameters, a single tube or a small set of tubes is run under cycling conditions that inherently favor amplification of the desired amplicon, often to the exclusion of artifactual amplicons and primer-dimers. Multiple cycles are programmed so that the annealing segments in sequential cycles are run at incrementally lower temperatures (see below). As cycling progresses, the annealing segment temperature, which was selected to be initially above the suspected $T_{\mathrm{m}}$, gradually declines to, and falls below, this level. This strategy helps ensure that the first primer-template hybridization events involve only those reactants with the greatest complementarity, (i.e., those yielding the target amplicon). Even though the an- 
nealing temperature may eventually drop down to the $T_{\mathrm{m}}$ of nonspecific hybridizations, the target amplicon already will have begun its geometric amplification and is thus in a position to outcompete any lagging (nonspecific) PCR products during the remaining cycles. Because the aim is to avoid low $T_{\mathrm{m}}$ priming during the earlier cycles, it is imperative that the hot start modification ${ }^{(13-15)}$ be utilized with TD PCR. TD PCR should be viewed not as a method of determining the optimum cycling conditions for a specific PCR but as a potential one-step method for approaching optimal amplification. We have found that a variety of otherwise satisfactory single amplicon-yielding reactions are rendered more robust (i.e., yield more product) when subjected to TD PCR (K.H. Roux and K. Hecker, unpubl.).

TD PCR is of particular value when the degree of identity between the primer and template is unknown. ${ }^{(16)}$ This situation often arises as primers are designed on the basis of amino acid sequences, members of a multigene family are amplified, or evolutionary PCR is attempted (i.e., amplification of DNA from one species using primers with identity to a homologous segment of another species). In such cases, the mismatches among the primers and template may have lowered the $T_{\mathrm{m}} \mathrm{s}$ of the target amplicons enough to approach those of the spurious priming sites. Degenerate primers with multiple base variation or inosine residues are often used in such situations, ${ }^{(17-21)}$ but the greater variety of sequences in the former case and the relaxed stringency in the latter case might tend to increase the chances of nonspecific priming. Moreover, in some cases the locations of potential base mismatches will be unknown. Although TD PCR can be used with degenerate primers, ${ }^{(17)}$ we have shown that nondegenerate primers displaying a significant degree of template-sequence mismatches can yield single-target amplicons of singlecopy genes from genomic DNA under standard buffer conditions. ${ }^{(16)}$ Even mismatches clustered near the $3^{\prime}$ end of the primer are tolerated.

\section{NESTED PCR}

Nested and seminested PCRs are often quite successful in reducing or eliminating unwanted products while at the same time dramatically increasing sensitivity. ${ }^{(14,22-25)}$ An initial set of primers straddling the DNA segment of interest is first amplified under standard conditions. Spurious products are frequently primed with one or both primers and contain irrelevant sequences internally. An aliquot of the reaction product mixture is then subjected to an additional round of amplification using primers complementary to the sequences internal to the first set of primers. Only the legitimate product should be amplified in this second round. This approach is often successful even if the desired product is initially below the level of detection by ethidium bromide staining and in the presence of visible spurious bands. Seminested PCR, in which a second primer is internal to only one end of the target segment, can be equally effective. ${ }^{(24)}$ This variation is often required for gene

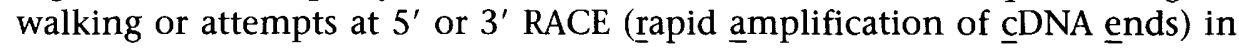
which the template DNA sequence internal to only one of the primers is known.

A second form of artifact, known as jumping PCR, may not be eliminated by nested PCR. Incompletely extended products can occasionally rehybridize to an adjacent segment of DNA, perhaps to a similar gene element, to prime an unintended product. ${ }^{(26)}$ In such instances, sequences internal to one or both primers will still be present, but the amplicon size will differ.

If it is anticipated that nested PCR methods will be employed, better results may be obtained if the first and second rounds of amplification are terminated after 20 or so cycles rather than the usual 30-35. This modification will minimize the chances of generating unwanted high molecular weight bands 
and smears. ${ }^{(9,24)}$ Such artifacts often contain considerable single-stranded DNA and appear to be the result of mispriming by DNA products amplified in earlier cycles. Nested PCR is extremely sensitive, and as little as a single copy of a viral gene has been detected in a background of $10^{6}$ genomes. ${ }^{(24)}$

\section{HOT START PCR}

Even brief incubations of a PCR reaction mix at temperatures significantly below the $T_{\mathrm{m}}$ can result in primer-dimer and nonspecific priming. Hot start PCR methods ${ }^{(13-15)}$ can dramatically reduce these problems. The aim is to withhold at least one of the critical components from participating in the reaction until the temperature in the first cycle rises above the $T_{\mathrm{m}}$ of the reactants. For example, in smaller assays incorporating an oil overlay, one of the components common to all tubes (e.g., Taq polymerase) can be initially withheld and added only after the temperature rises above $80^{\circ} \mathrm{C}$ during the first denaturing stage. Alternatively, a wax bead can be melted over the bulk of the reaction mix in each tube and allowed to solidify and the withheld component pipetted on top of the wax cap. These beads can be made in the laboratory ${ }^{(27,28)}$ or purchased (Ampliwax PCR Gems, Perkin-Elmer Cetus). During the temperature ramp into the first denaturation segment, the wax will melt and the final component will become incorporated and mixed by convection in each tube, a great convenience in dealing with large numbers of tubes. A recent hot start variation involves adding specific anti-Taq polymerase antibody (TaqStart Antibodies, Clontech) to the PCR reaction tubes prior to the addition of Taq polymerase. The antibody prevents polymerase activity from beginning until the rising temperature dissociates and denatures the blocking antibody. This modification is compatible with newer thermal cyclers and techniques that seek to avoid the extra handling and purification steps accompanying oil and wax overlays.

\section{CONTROLS}

Negative controls are mandatory in each PCR run to rule out false-positives caused by contamination. All primer sets and each new aliquot of buffer component must be tested. Fortunately, often this can be done in one or two tubes by incorporating all of the reagents except the DNA template. Even if multiple primer sets are being used, all can be run in the same tube. It is often desirable to run positive controls as well to ensure that master mixes have been formulated properly and to aid in optimization. When amplification from genomic DNA or some other low copy number source is attempted, it may be advisable first to optimize with higher-copy dilutions $\left(10^{3}-10^{5}\right.$ copies $)$ of the target DNA segment from a plasmid or phage prep if available. Of course, great care must be exercised not to create contamination problems in the process. Another useful control is to purify irrelevant DNA in parallel with the template-containing DNA. PCR of this template-free DNA will serve as a process control to monitor for contamination picked up during the purification procedure.

\section{CONTAMINATION}

The best way to deal with the ever-present threat of contamination is to take every precaution to prevent it from happening in the first place. ${ }^{(29)}$ If the intended target template is from a human source or a common laboratory vector, the chance of contamination is considerably increased. All reagents including primers, templates, and $\mathrm{H}_{2} \mathrm{O}$ should be stored in multiple small (ideally, single-use) aliquots and freely discarded if suspected of contamination. The use of positive displacement micropipettes and tips or standard 
micropipettes with filter-plugged tips may be advantageous. Pipetting should be slow and deliberate to minimize the generation of aerosols. Hands should be gloved at all times, and gloves should be changed frequently. A likely source of much contamination occurs when reaction tubes are opened and closed and can take the form of aerosol generation or inadvertent contact with the inner lip of the cap. Loose-fitting gloves tend to aggravate the problem. Tube-opening devices may be useful but can themselves be sources of contamination. Contamination also can be introduced during the purification of the template through equipment and reagents (e.g., homogenizers, reused tube, electrophoresis apparatus, centrifuges, common laboratory buffers, and phenol).

The primary source of contamination in many laboratories is carryover from previous PCRs. It is prudent to keep the workstations, including the pipettes, racks, and reagents used for setting up PCR physically separate from those used for analyzing the reactions (in separate rooms if possible). A particular dilemma arises when secondary amplification is required (i.e., additional cycles are needed, a reamplification of a previous reaction is desired, or two-step protocols such as nested PCR are conducted). In such cases, sterile, uncapped tubes containing all of the reactants except the diluted template and Taq polymerase (withheld for hot start) are placed on a freshly prepared "sterile" field (i.e., a fresh sheet of plastic wrap or foil) to receive an aliquot of the first amplification product. Care is taken not to touch the tubes or rack with anything (including gloved hands) but the pipette tip during the transfer process. With a fresh pair of gloves, the tubes and rack can be transferred to the thermal cycler for further processing.

No matter how much care is exercised, contamination may occur on occasion. Several methods for dealing with this eventuality have been described. Environmental surfaces, including pipettes, can be decontaminated by wetting with a $0.07 \mathrm{M}$ sodium hypochlorite (i.e., $10 \%$ Clorox bleach) solution. ${ }^{(30)}$ This method has been found to be more efficient and less corrosive than decontamination with $\mathrm{HCl}$. UV irradiation of the work station is also beneficial, though dried DNA is much less susceptible to UV damage than hydrated forms. Containers, racks, and micropipettors, if permissible, should be autoclaved. Also, reagents can be decontaminated with UV light. ${ }^{(31,32)}$ The various components of a reaction mix display dramatically different sensitivities to UV-induced irreversible denaturation. ${ }^{(33)}$ Because dNTPs in the concentrations used in PCR are strong absorbers of UV irradiation and can effectively block decontamination efforts of final reaction mixes, it is advisable to decontaminate individual stock reagents or at least to withhold the dNTPs (perhaps in conjunction with hot start) until after reaction mix decontamination. Primers vary widely in their susceptibility to UV-induced denaturation depending, in part, on the likelihood of thymine dimer formation. $\mathrm{MgCl}_{2}$, stock $10 \times$ buffers, and dNTPs are UV insensitive, whereas Taq polymerase is UV sensitive. ${ }^{(33)}$ The nature of the contaminant also influences the effectiveness of decontamination efforts. DNA of $<250$ bases is neutralized less effectively. To decontaminate, place reaction components, less Taq polymerase and template, in clear reaction tubes on a Fotodyne 1000 transilluminator containing 254- and 300-nm UV bulbs or Photodyne Foto/Prep I (without transparent cover) with 300-nm bulbs or similar devices for 10-20 min. The 300-nm irradiation alone can be used but is somewhat less effective. A 10-min treatment in a Stratalinker 1800 (Stratagene) set to deliver $100 \mathrm{~mJ}$ per min at $254 \mathrm{~nm}$ can also be used. ${ }^{(2)}$

Other approaches to decontamination include $\gamma$ irradiation, ${ }^{(34)}$ digestion with restriction endonucleases, ${ }^{(35,36)}$ DNaseI, $^{(35)}$ exonuclease III, ${ }^{(37)}$ isopsoralen treatment, ${ }^{(38,39)}$ incorporation of $\mathrm{dU}$ into PCR products followed by uracil- $N$-glycosylase (UNG) digestion, ${ }^{(40)}$ and centrifugal ultrafiltration. ${ }^{(41)}$ 
None of these additional approaches is ideal. An appropriate $\gamma$-ray source is frequently not available. Enzymatic digestions usually entail additional manipulations, which increase the risk of contamination.

The last two approaches are primarily directed at treating PCR products so as to render them incapable of serving as templates (i.e., preventing amplicons from serving as sources of carryover contamination for future PCRs). Isopsoralen derivatives are thermostable molecules capable of forming polymerase-inhibiting monoadducts upon photoactivation in the 320- to 400-nm light range. Isopsoralens can be conveniently added during PCR setup and subsequently activated by UV exposure to sterilize PCR products after amplification but before tube opening. ${ }^{(38,39)}$ The amount of isopsoralen added per tube depends on experimental conditions.

Taq polymerase readily incorporates uracil in place of thymine into PCR amplicons. As a consequence, UNG can be used to digest previously amplified uracil-containing amplicons that might otherwise be contaminating a fresh PCR reaction mix. ${ }^{(40)}$ UNG depyrimidinates uracil residues to block polymer extension and increase the rate of strand hydrolysis. UNG is not reactive with free dUTP and is thermolabile. Consequently, $5 \mathrm{ng}$ of UNG can be added to a PCR mix and incubated for 15 min to digest uracil-containing DNA. The UNG is denatured during a 5 -min incubation at $94^{\circ} \mathrm{C}$ preceding a normal PCR cycling program. Kits and reagents for UNG decontamination are available from GIBCO-BRL and Perkin-Elmer Cetus. One drawback to these procedures is that neither isopsoralen nor UNG treatments will be effective against contamination originating from sources other than carryover from previous PCRs.

\section{NO PRODUCT}

You have adjusted the $\mathrm{Mg}^{2+}$ concentration, buffer $\mathrm{pH}$, and cycling parameters; added more cycles; tried lower annealing temperatures and TD PCR; and still no product is seen on ethidium bromide-stained gels (acrylamide gels are considerably more sensitive than agarose gels). What is the next step? Lengthening the initial denaturation step and/or increasing temperature will increase the likelihood that the template DNA is fully denatured to provide the maximal number of priming sites. Standard conditions for this optional step are $5 \mathrm{~min}$ at $95^{\circ} \mathrm{C}$. An in-tube thermocouple can be used to predetermine that the indicated temperature will correspond to the actual sample temperature.

Amplification may have occurred but may have been inefficient. If so, the amplicons can be revealed by a probe of the dried gel or a blot. A secondary amplification using the same primers or, preferably, nested primers may be all that is needed to generate a specific product. Serial 10-fold dilutions ranging from 1:100 to $1: 10,000$ should be used.

\section{INHIBITORS}

Poor or nonexistent amplification may indicate the presence of inhibitors in the DNA sample. Numerous inhibitors of PCR have been described. These include ionic detergents (e.g., SDS and Sarkosyl), ${ }^{(42)}$ phenol, heparin, ${ }^{(43)} \mathrm{xy}$ lene cyanol, and bromphenol blue. ${ }^{(4)}$ Test for inhibitor in the template preparation by spiking original PCR mix with dilutions of known positive (demonstrably amplifiable) template. Re-extraction, ethanol precipitation, and/or centrifugal ultrafiltration may resolve the problem. Proteinase K carryover can serve to digest the Taq polymerase but is readily denatured by a 5 -min incubation at $95^{\circ} \mathrm{C}$. 


\section{SUGGESTED OPTIMIZATION STRATEGY}

The example given is TD PCR, but the same principles apply to conventional PCR.

1. Design an optimal primer pair on the basis of considerations discussed above.

2. Calculate or estimate approximate $T_{\mathrm{m}}$. Program the thermal cycler for TD PCR as described below.

3. Set up several standard hot start PCR mixes incorporating a range of $\mathrm{Mg}^{2+}$ concentrations and including appropriate positive and negative controls. Use $10^{4}-10^{5}$ copies of the template.

4. Amplify and analyze products.

a. If weak or no product detected,

(1) Subject reaction tubes to 10 additional cycles at constant annealing temperature (i.e., $55^{\circ} \mathrm{C}$ ) and recheck.

(2) Reamplify $1 \mu$ l of 10 -fold dilutions $(1: 10-1: 1,000)$ of TD PCR at fixed annealing temperature.

(3) Use more template and check for inhibitor in template preparation by spiking original PCR mix with dilutions of known positive (demonstrably amplifiable) template.

(4) Increase initial template denaturation time and/or temperature.

(5) Vary concentrations of other buffer components ( $\mathrm{pH}$, Taq polymerase, dNTPs, primers).

(6) Add enhancers to PCR mix.

(7) Reamplify dilutions $(1: 10-1: 1,000)$ of first reaction using nested primers.

(8) Abandon this primer set, design new primers, and begin again. Depending on the researcher's degree of impatience and tolerance for frustration, this step might supercede any of the above.

b. If multiple products or a high molecular weight smear is observed,

(1) Raise the maximum and minimum annealing temperatures (i.e., move the range upward) in the TD PCR program.

(2) Remove some cycles from the bottom of the range and/or from the terminal constant temperature cycles.

(3) Increase the number of cycles per degree annealing temperature by one cycle (i.e., to three cycles/degree). Doing so may necessitate removing terminal cycles to prevent smearing.

(4) Vary concentrations of other buffer components ( $\mathrm{pH}, \mathrm{Taq}$ polymerase, dNTPs, primers).

(5) Attempt band purification followed by reamplification. Target bands can be cut from gels and allowed to diffuse out or be liberated by freeze/thaw cycles or gel digestion. Alternatively, a small plug of gel can be removed with a micropipette tip, or most simply, by stabbing the band directly in the gel with an autoclaved toothpick (plastic may be preferable) ${ }^{(45)}$ and inoculating a fresh reaction tube.

(6) Reamplify $1: 10^{4}$ and $1: 10^{5}$ dilutions of first reaction using nested primers.

(7) Abandon primer set, design new primers, and begin again.

\section{TOUCHDOWN PCR PROGRAMMING}

The goal in programming for TD PCR is to produce a series of cycles with progressively lower annealing temperatures. The annealing temperature range should span $\sim 15^{\circ} \mathrm{C}$ and extend from at least a few degrees above the estimated $T_{\mathrm{m}}$ to $\sim 10^{\circ} \mathrm{C}$ below. For example, for a calculated primer template $T_{\mathrm{m}}$ of $62^{\circ} \mathrm{C}$ with no degeneracy, program the thermal cycler to decrease the annealing temperature $1^{\circ} \mathrm{C}$ every second cycle (i.e., run two cycles per degree) 
from $65^{\circ} \mathrm{C}$ to $50^{\circ} \mathrm{C}$, followed by 15 additional cycles at $50^{\circ} \mathrm{C}$. Some thermal cyclers (Perkin-Elmer model 9600 and MJ Research model PTC-100) readily accommodate TD PCR and are easily programmed to decrease the temperature of a segment automatically by a fixed amount per cycle (e.g., $0.5^{\circ} \mathrm{C}$ / cycle). For others, a long series of files must be linked or extensive strings of commands entered. In these latter cases, it may be more convenient to create a generic TD PCR program covering a broader temperature range $\left(\sim 20^{\circ} \mathrm{C}\right)$ than to reprogram every time the range needs to be modified by a few degrees. Another alternative to programming restrictions and inconvenience is to use fewer but more abrupt steps (e.g., seven $2^{\circ} \mathrm{C}$ steps, or five $3^{\circ} \mathrm{C}$ steps); doing so may decrease the chances for discriminating among products with two closely spaced $T_{\mathrm{m}} \mathrm{s}$.

The continued presence of spurious bands following TD PCR indicates that the initial annealing temperature was too low, that there is a relatively small gap between the $T_{\mathrm{m}} \mathrm{s}$ of the target and unwanted amplicons and/or that the unwanted amplicon is being more efficiently amplified. Raising the number of cycles per $1^{\circ} \mathrm{C}$ descending step to three or four will give the target amplicon added competitive advantage before the initiation of the spurious amplification. A proportional number of cycles should be removed from the end of the program to prevent excess cycling and the concomitant degradation of the amplicon and generation of high molecular weight smears.

Modifications of TD PCR for use with degenerate and mismatched primers include lowering the annealing temperature range (e.g., $50^{\circ} \mathrm{C}$ declining to $35^{\circ} \mathrm{C}$ ) while keeping the last 15 cycles at $>50^{\circ} \mathrm{C}$ (once priming has begun, the primers are fully complementary to the newly formed amplicons, have a much higher $T_{\mathrm{m}}$, and do not benefit from excessively low annealing temperatures).

\section{CONDITIONS FAVORING ENHANCED SPECIFICITY}

Adjusting conditions in the direction opposite to that listed below usually favors increased sensitivity (i.e., more product) and the concomitant risk of nonspecific amplification. The aim is to strike a balance between these two opposing tendencies. ( $\uparrow$ and $\downarrow$ signify increase and decrease, respectively).

Use hot start

Use TD PCR (favors enhancer specificity and sensitivity)

Optimize primer design

$\downarrow \mathrm{Mg}^{2+}$

$\downarrow$ dNTP (also favors higher fidelity)

Optimize $\mathrm{pH}$

$\downarrow$ Taq polymerase

$\downarrow$ cycle segment lengths

$\downarrow$ number of cycles

$\uparrow$ annealing temperature

$\downarrow$ inhibitors

$\uparrow$ ramp speed

$\uparrow$ chance that target temperature is achieved in each tube

Add and optimize enhancer(s)

$\downarrow$ primer concentration

$\downarrow$ primer degeneracy

$\uparrow$ template denaturation efficiency

\section{ACKNOWLEDGMENTS}

I thank Rani Dhanarajan, Dan Garza, and Karl Hecker for their valuable comments. 


\section{REFERENCES}

1. Pomp, D. and J.F. Medrano. 1991. Organic solvents as facilitators of polymerase chain reaction. BioTechniques 10: 58-59.

2. Newton, C.R. and A. Graham. 1994. PCR. Bios Scientific Publishers, Oxford, England.

3. Taguchi, G. 1986. Introduction to quality engineering. In Asian productivity organisation UNIPUB, New York, New York.

4. Cobb, B.D. and J.M. Clarkson. 1994. A simple procedure for optimizing the polymerase chain reaction (PCR) using modified Taguchi methods. Nucleic Acids Res. 22: 3801-3805.

5. Lowe, T., J. Sharefkin, S.Q. Yang, and C.W. Diefenbach. 1990. A computer program for selection of oligonucleotide primers for polymerase chain reactions. Nucleic Acids Res. 18: 1757-1761.

6. Rychlik, W. and R.D. Rhoads. 1989. A computer program for choosing optimal oligonucleotides for filter hybridation, sequencing, and in vitro amplification of DNA. Nucleic Acids Res. 17: 8543-8549.

7. He, Q., M. Marjamaki, H. Soini, J. Mertsola, and M.K. Viljanen. 1994. Primers are decisive for sensitivity of PCR. BioTechniques 17: 82-87.

8. Sharrocks, A. D. 1994. The design of primers for PCR. In PCR technology: Current innovations. (ed. H.G. Griffin and A.M. Griffin), pp. 5-11. CRC Press, Boca Raton, FL.

9. Bell, D.A. and D. DeMarini. 1991. Excessive cycling converts PCR products to random-length higher molecular weight fragments. Nucleic Acids Res. 19: 5079.

10. Linz, U. 1990. Thermocycler temperature variation invalidates PCR results. BioTechniques 9: 286-292.

11. Hoelzel, R. 1990. The trouble with "PCR" machines. Trends Genet. 6: 237-238.

12. Don, R.H., P.T. Cox, B.J. Wainwright, K. Baker, and J.S. Mattick. 1991. "Touchdown" PCR to circumvent spurious priming during gene amplification. Nucleic Acids Res. 19: 4008.

13. Erlich, H.A., D. Gelfand, and J.J. Sninsky. 1991. Recent advances in the polymerase chain reaction. Science 252: 1643-1651.

14. Mullis, K.B. 1991. The polymerase chain reaction in an anemic mode: How to avoid cold oligodeoxyribonuclear fusion. PCR Methods Applic. 1: 1-4.

15. D'Aquila, R.T., L.J. Bechtel, J.A. Viteler, J.J. Eron, P. Gorczyca, and J.C. Kaplin. 1991. Maximizing sensitivity and specificity of PCR by preamplification heating. Nucleic Acids Res. 19: 3749.

16. Roux, K.H. 1994. Using mismatched primer-template pairs in touchdown PCR. BioTechniques 16: 812-814.

17. Batzer, M.A., J.E. Carlton, and P.L. Deininger. 1991. Enhanced evolutionary PCR using oligonucleotides with inosine at the 3 '-terminus. Nucleic Acids Res. 19: 5081.

18. Knoth, K., S. Roberds, C. Poteet, and M. Tamkun. 1988. Highly degenerate inosine-containing primers specifically amplify rare cDNA using the polymerase chain reaction. Nucleic Acids Res. 16: 10932.

19. Lee, C., X. Wu, R.A. Gibbs, R.G. Cook, D.M. Muzny, and C.T. Caskey. 1988. Generation of cDNA probes directed by amino acid sequence: Cloning of urate oxidase. Science 239: 12881291.

20. Patil, R.V. and E.E. Dekker. 1990. PCR amplification of an Escherichia coli gene using mixed primers containing deoxyinosine at ambiguous positions in degenerate amino acid codons. Nucleic Acids Res. 18: 3080.

21. Peterson, M.G., J. Inostroza, M.E. Maxon, O. Flores, A. Adomon, D. Reinberg, and R. Tjian. 1991. Structure and functional properties of human general transcription factor IIE. Nature 354: 369-373.

22. Mullis, K. and F.A. Faloona. 1987. Specific synthesis of DNA in vitro via a polymerase-catalyzed chain reaction. Methods Enzymol. 155: 335-350.

23. Gibbs, R.A. 1990. DNA amplification by the polymerase chain reaction. Anal. Chem. 62: 1202-1214.

24. Zhang, X-Y. and M. Ehrlich. 1994. Detection and quantitation of low numbers of chromosomes containing bcl-2 oncogene translocations using semi-nested PCR. BioTechniques 16: 502-507.

25. Zimmermann, K., K. Pischinger, and J.W. Mannhalter. 1994. Nested primer PCR detection limits of HIV-1 in a background of increasing numbers of lysed cells. BioTechniques 17: 18-20.

26. Huang, L.-M. and K.-T. Jeang. 1994. Long-range jumping of incompletely extended polymerase chain fragments generates unexpected products. BioTechniques 16: 242-246.

27. Wainwright, L.A. and H.S. Seifert. 1993. Paraffin beads can replace mineral oil as an evaporation barrier in PCR. BioTechniques 14: 34-36.

28. Bassam B.J. and G. Caetano-Anolles. 1993. Automated "hot start" PCR using mineral oil and paraffin wax. BioTechniques 14: 30-34.

29. Kwok, S. and R. Higuchi. 1989. Avoiding false positives with PCR. Nature 339: 237-238.

30. Prince, A.M. and L. Andrus. 1992. PCR: How to kill unwanted DNA. BioTechniques 12: 358360. 
31. Sakar, G. and S. Sommer. 1990. More light on PCR contamination. Nature 347: 340-341.

32. Ou, C.-Y., J.L. Moore, and G. Schochetman. 1991. Use of UV irradiation to reduce false positivity in polymerase chain reaction. BioTechniques 10: $442-445$.

33. Frothingham, R., R.B. Blitchington, D.H. Lee, R.C. Greene, and K.H. Wilson. 1992. UV absorption complicates PCR decontamination. BioTechniques 13: 208-210.

34. Deragon, J.-M., D. Sinnett, G. Mitchell, M. Potier, and D. Labuda. 1990. Use of $\gamma$-irradiation to eliminate DNA contamination for PCR. Nucleic Acids Res. 18: 6149.

35. Furrer, B., U. Candrian, P. Wieland, and J. Luthy. 1990. Improving PCR efficiency. Nature 346: 324.

36. DeFilippes, F.M. 1991. Decontaminating the polymerase chain reaction. BioTechniques 10: 26-30.

37. Zhu, Y.S., S.T. Isaacs, G.D. Cimino, and J.E. Hearst. 1991. The use of exonuclease III for polymerase chain reaction sterilization. Nucleic Acids Res. 19: 2511.

38. Cimino, G.D., K.C. Metchette, J.W. Tessman, J.E. Hearst, and S.T. Isaacs. 1990. Post-PCR sterilization: A method to control carryover contamination for the polymerase chain reaction. Nucleic Acids Res. 19: 99-107.

39. Isaacs, S.T., J.W. Tessman, K.C. Metchette, J.E. Hearst, and G.D. Cimino. 1990. Post-PCR sterilization: Development and application to HIV-1 diagnostic assay. Nucleic Acids Res. 19: 109-116.

40. Longo, M.C., M.S. Berninger, and J.L. Hartley. 1990. Use of uracil DNA glycosylase to control carry-over contamination in polymerase chain reactions. Gene 93: 125-128.

41. Krowczynska, A.M. and M.B. Henderson. 1992. Efficient purification of PCR products using ultrafiltration. BioTechniques 13: 286-289.

42. Weyant, R.S., P. Edmonds, and B. Swaminathan. 1990. Effect of ionic and nonionic detergents on the Taq polymerase. BioTechniques 9: 308-309.

43. Beutler, E., T. Gelbart, and W. Kuhl. 1990. Interference of heparin with the polymerase chain reaction. BioTechniques 9: 166.

44. Hoppe, B.L., B.M. Conti-Tronconi, and R.M. Horton. 1992. Gel-loading dyes compatible with PCR. BioTechniques 12: 679-680.

45. Lee, A.B. and T.A. Cooper. 1995. Improved direct PCR screen for bacterial colonies: Wooden toothpicks inhibit PCR amplification. BioTechniques 18: 225-226. 


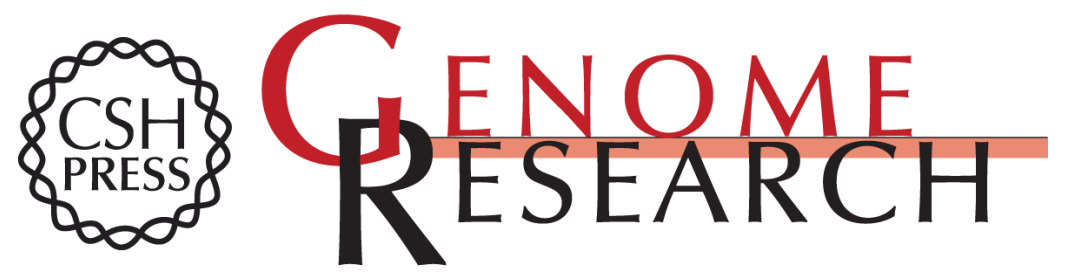

\section{Optimization and troubleshooting in PCR.}

K H Roux

Genome Res. 1995 4: S185-S194

References This article cites 42 articles, 2 of which can be accessed free at: http://genome.cshlp.org/content/4/5/S185.full.html\#ref-list-1

\section{License}

Email Alerting Receive free email alerts when new articles cite this article - sign up in the box at the Service top right corner of the article or click here.

\section{Affordable, Accurate Sequencing.}

To subscribe to Genome Research go to: https://genome.cshlp.org/subscriptions 